\title{
Application of Four-Dimension Assignment Algorithm of Data Association in Distributed Passive-Sensor System
}

\author{
Li Zhou ${ }^{1,2}$, You He ${ }^{1}$, and Xiao-jing Wang ${ }^{3}$ \\ ${ }^{1}$ Research Institute of Information Fusion, Naval Aeronautical, \\ Engineering Institute, Yantai, 264001, P. R. China \\ zxyzlzwh@vip.sina.com \\ 2 Math \& Information College, Yantai Teachers' University, \\ Yantai, 264025, P. R. China \\ ${ }^{3}$ Department of Basic Science, \\ Beijing Institute of Civil Engineering and Architecture, \\ Beijing, 100044, P.R. China
}

\begin{abstract}
The disadvantage of multi-dimension assignment algorithm of data association in distributed passive-sensor system is the lower processing speed. The modified optimal assignment algorithm is presented in this paper. The new algorithm avoids a large quantity of calculation of cost function of 4-tuple measurements and removes the disturbance of some false location points. Simulation results show that by using the algorithm discussed in this paper, not only the calculation burden is reduced greatly but also the accuracy of data association is improved correspondingly.
\end{abstract}

\section{Introduction}

Because passive-sensor doesn't radiate any electromagnetism signal, and has the advantage of both better concealment and less interference of the enemy, passivesensor location has become a hot research point which is studied by many scholars. When the target's position is estimated by bearing-only measurements from passive sensors, the better method of data association is to transform the problem to the optimal assignment problem of operational research [1], [2]. The disadvantage of the optimal assignment algorithm of multi-passive-sensor multi-target data association is the long processing time caused by the heavier calculation burden of cost function [3]. In the case of the four bearing-only sensors and multi-target are in the same plane, this paper proposes a modified algorithm based on direction-finding cross location. The essence of the modified algorithm is a two-stage association algorithm. In the first stage, we eliminate some false intersection points by using a cross location technology, and in the second stage, only those points which have passed through the gating of the correlation test in the first stage can be permitted to join the assignment process. This reduces the computation burden from the calculation of cost function for assignment problem. In the meantime, with the removing of the large number of false location points, the effect of data association is improved. 


\section{Data Association}

\subsection{The Traditional Optimal Algorithm}

Supposed four bearing-only sensors are used to locate targets as illustrated in Fig.1. The positions of four sensors are $\boldsymbol{p}_{s}=\left(x_{s}, y_{s}\right)^{T}, \quad s=1,2,3,4$; suppose there are $N$ targets in Surveillance view, and the position of target $t$ is $\boldsymbol{p}_{t}=\left(x_{t}, y_{t}\right)^{T}$; Suppose the number of measurements of sensor $\mathrm{s}$ is $n_{s}, s=1,2,3,4$; the bearing-only sensor only measures the azimuth of target $t$, and it is denoted as $\boldsymbol{Z}_{s i_{s}}, \quad i_{s}=1,2 \ldots n_{s}$; if the measurement is from a real target, it is the true observable $\theta_{s t}$ plus the Gaussian noise $N\left(0, \sigma_{s}^{2}\right)$, and if it is from spurious measurement, suppose it follows the uniform distribution in the field of view of sensor $s$.

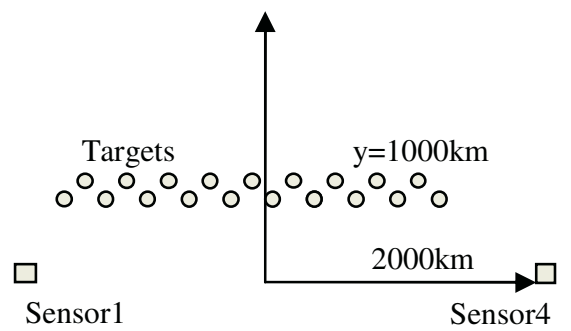

Sensor2 Sensor3

Fig. 1. Position of sensors

The maximum likelihood function of 4-tuple $Z_{i_{1} i_{2} i_{3} i_{4}}=\left\{Z_{1 i_{1},} Z_{2 i_{2}}, Z_{3 i_{3}}, Z_{4 i_{4}}\right\}$ coming $\mathrm{f}^{\text {rom the same target } t \text { is [1], [2] }}$

$$
\Lambda\left(\boldsymbol{Z}_{i_{1} i_{2} i_{3} i_{s}} \mid \boldsymbol{\omega}_{t}\right)=\prod_{s=1}^{4}\left[P_{d s} \cdot p\left(\boldsymbol{Z}_{s i_{s}} \mid \boldsymbol{\omega}_{t}\right)\right]^{u\left(i_{s}\right)}\left[1-p_{d s}\right]^{\left[1-u\left(i_{s}\right)\right]}
$$

Where $P_{d s}$ is the detect probability of sensor $s, u\left(i_{s}\right)$ is a binary indicator function, if $s_{s}$ ensor $\mathrm{s}$ missed the detection from target $t$, i.e., $i_{s}=0$, then $u\left(i_{s}\right)=0$. Otherwise, $u\left(i_{s}\right)=1 . p\left(\boldsymbol{Z}_{s i_{s}} \mid \boldsymbol{\omega}_{t}\right)$ is the probability density function of $\boldsymbol{Z}_{s i_{s}}$ being from target $t$, The likelihood that the measurements are all spurious or unrelated to target $t$, i.e., $t=\boldsymbol{\Phi}$ is 


$$
\Lambda\left(\boldsymbol{Z}_{i_{1} i_{2} i_{3} i_{4}} \mid t=\boldsymbol{\Phi}\right)=\prod_{s=1}^{4}\left[\frac{1}{\boldsymbol{\Psi}_{s}}\right]^{u\left(i_{s}\right)}
$$

where $\boldsymbol{\Psi}_{s}$ is the field of view of sensor $s$, the cost of associating the 4-tuple to target $t$ is given by

$$
c_{i_{1} i_{2} i_{3} i_{4}}=-\ln \frac{\Lambda\left(Z_{i_{1} i_{2} i_{3} i_{4}} \mid t\right)}{\Lambda\left(Z_{i_{1} i_{2} i_{3} i_{4}} \mid t=\mathbf{\Phi}\right)}
$$

As $\omega_{t}$ in (1) is unknown, it can usually be replaced by its maximum likelihood or least-square estimation as

$$
\hat{\boldsymbol{\omega}_{t}}=\arg \max _{\boldsymbol{\omega}_{t}} \Lambda\left(\boldsymbol{Z}_{i_{1} i_{2} i_{3} i_{4}} \mid t\right)
$$

Hence, the cost of associating $\boldsymbol{Z}_{i_{1} i_{2} i_{3} i_{4}}$ with target $t$ can be induced as [1], [2]

$$
\begin{aligned}
c_{i_{1} i_{2} i_{3} i_{4}} & =\sum_{s=1}^{4}\left[u ( i _ { s } ) \left(\ln \left(\frac{\sqrt{2 \pi} \cdot \sigma_{s}}{P_{d s} \cdot \boldsymbol{\Psi}_{s}}\right)\right.\right. \\
& \left.\left.+\frac{1}{2}\left(\frac{\boldsymbol{Z}_{s i_{s}}-\hat{\theta_{s t}}}{\sigma_{s}}\right)^{2}\right)-\left(1-u\left(i_{s}\right)\right) \cdot \ln \left(1-P_{d s}\right)\right]
\end{aligned}
$$

With the assumption that each measurement is assigned to a target or declared false, and each measurement is assigned to at most one target, the problem of data association can be transformed to the following generalized 4-D assignment problem [2]

$$
\min _{\rho_{i} i_{2} i_{3} i_{4}} \sum_{i_{1}=0}^{n_{1}} \sum_{i_{2}=0}^{n_{2}} \sum_{i_{3}=0}^{n_{3}} \sum_{i_{4}}^{n_{4}} c_{i_{1} i_{2} i_{3} i_{4}} . \rho_{i_{1} i_{2} i_{3} i_{4}}
$$

subject to

$$
\begin{cases}\sum_{i_{2}=0}^{n_{2}} \sum_{i_{3}=0}^{n_{3}} \sum_{i_{4}=0}^{n_{4}} \rho_{i_{1} i_{2} i_{3} i_{4}}=1 ; & \forall i_{1}=1,2 \cdots n_{1} \\ \sum_{i_{1}=0}^{n_{1}} \sum_{i_{3}=0}^{n_{3}} \sum_{i_{4}=0}^{n_{4}} \rho_{i_{1} i_{2} i_{3} i_{4}}=1 ; & \forall i_{2}=1,2 \cdots n_{2} \\ \sum_{i_{1}=0}^{n_{1}} \sum_{i_{2}}^{n_{2}} \sum_{i_{4}}^{n_{4}} \rho_{i_{1} i_{2} i_{3} i_{4}}=1 ; & \forall i_{3}=1,2 \ldots n_{3} \\ \sum_{i_{1}}^{n_{1}} \sum_{i_{2}=0}^{n_{2}} \sum_{i_{3}=0}^{n_{3}} \rho_{i_{1} i_{2} i_{3} i_{4}}=1 ; & \forall i_{4}=1,2 \ldots n_{4}\end{cases}
$$

The solution of 4-D assignment problem can be shown to be NP-hard [2], [5]. The optimal technique requires unacceptable time and is of little practical value. Instead, fast and near the optimal solutions are most desirable. Among various heuristic 
algorithms of multi-dimension assignment problem, Lagrangian relaxation algorithm has a dominant role owing to its satisfying result in application. It relaxes the 4-D assignment problem to a series of 2-D assignment problem to solve, which can be resolved by various algorithms in polynomial time [4]. The advantage of this algorithm compared with the other modern optimal algorithm is that not only can we obtain a suboptimal solution which is near to the optimal solution, but also can obtain a measure of the quality of this solution. When the density of targets and false alarms in surveillance is higher, the run time of Lagrangian relaxation algorithm is still too long to satisfy the need of engineering because of the large quantity of calculation burden from association cost, so we present a modified algorithm in next section.

\subsection{The Modified Algorithm}

The description of the cross location for four bearing-only sensors are shown in Fig.2. $\theta_{i}(i=1,2,3,4)$ denote the bearing measurements, The positions of four sensors are denoted by $\left(x_{\mathrm{i}}, y_{\mathrm{i}}\right), i=1,2,3,4$, and the Cartesian position of A, B,C is denoted by $\left(x_{\mathrm{A}}\right.$, $\left.y_{\mathrm{A}}\right),\left(x_{\mathrm{B}}, y_{\mathrm{B}}\right),\left(x_{\mathrm{C}}, y_{\mathrm{C}}\right)$. Then from the formula

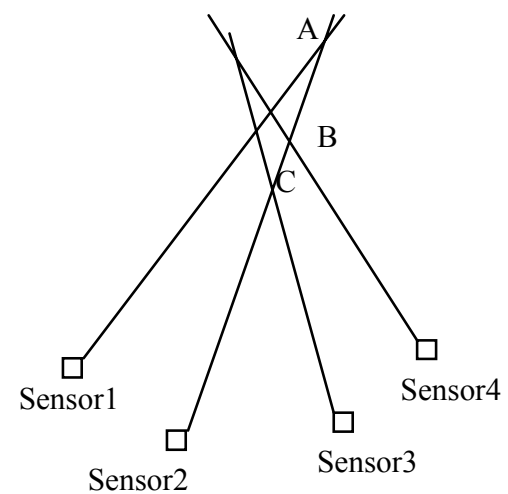

Fig. 2. Location of four sensors

$$
\operatorname{tg} \theta_{1}=\frac{y_{A}-y_{1}}{x_{A}-x_{1}}, \operatorname{tg} \theta_{2}=\frac{y_{A}-y_{2}}{x_{A}-x_{2}}
$$

We can get [3],

$$
\begin{aligned}
& x_{A}=\frac{y_{2}-y_{1}+x_{1} \operatorname{tg} \theta_{1}-x_{2} \operatorname{tg} \theta_{2}}{\operatorname{tg} \theta_{1}-\operatorname{tg} \theta_{2}} \\
& y_{A}=\frac{y_{2} \operatorname{tg} \theta_{1}-y_{1} \operatorname{tg} \theta_{2}+\left(x_{1}-x_{2}\right) \operatorname{tg} \theta_{1} \operatorname{tg} \theta_{2}}{\operatorname{tg} \theta_{1}-\operatorname{tg} \theta_{2}}
\end{aligned}
$$

Let

$$
d_{A B}=\sqrt{\left(x_{B}-x_{A}\right)^{2}+\left(y_{B}-y_{A}\right)^{2}}
$$


Again from

$$
\operatorname{tg} \theta_{2}=\frac{y_{B}-y_{2}}{x_{B}-x_{2}}, \operatorname{tg} \theta_{3}=\frac{y_{B}-y_{3}}{x_{B}-x_{3}},
$$

and

$$
\operatorname{tg} \theta_{3}=\frac{y_{C}-y_{3}}{x_{C}-x_{3}}, \operatorname{tg} \theta_{4}=\frac{y_{C}-y_{3}}{x_{C}-x_{3}}
$$

we can get the Cartesian position of $\mathrm{B}, \mathrm{C}$ and the distance function $d_{B C}$ and $d_{A C}$ in the same way.

Let

$$
d=d_{A B}+d_{B C}+d_{A C}
$$

In actuality, the Cartesian position of A, B and C usually don't coincide because of the sensor's measurement error. In consideration of the special position of sensor 2, the probability that the four measurements come from a same target can be estimated by the value of $d$. In general, the smaller the value is, the more possible that they come from a same target has. We can use the value of $d$ to be the statistics to determine if we should calculate the association cost of 4-tuple of measurements. This can avoid the large operation quantity from statistic test aiming to each 4-tuple of measurements one by one. The disadvantages using distance function to construct test statistics of $\chi^{2}$ distribution to carry on the data correlation test, one is the heavy burden of calculation, the other is inferior data association result caused by the reason that the statistic may be not obey to the $\chi^{2}$ distribution strictly sometimes. According to the large number of numeral simulations with the various measurement errors, the gating of statistics $d$ can be determined by $\tau=500 \times 180 \times \sigma / \pi$, where $\sigma$ denotes the bearing measurement error of each sensor, if it is different from various sensors, $\sigma$ can be replaced by the average of four error values.

As the association cost of 4-D assignment problem is defined as formula (3), so the corresponding 4-tuple of measurements $\boldsymbol{Z}_{i_{1} i_{2} i_{3} i_{4}}$ may be considered a candidate association if and only if $c_{i_{1} i_{2} i_{3} i_{4}}<0$, and all the 4-tuple of measurements $Z_{i_{1} i_{2} i_{3} i_{4}}$ with $c_{i_{1} i_{2} i_{3} i_{4}}>0$ can be eliminated from the list of candidate associations by a certain measure. The sparsity of candidate association is defined as the ratio of the number of potential measurement-target association in the 4-D assignment problem to the number of a fully connection. In the former algorithm, sparsity $s_{0}$ is defined as the ratio of the number of association with negative cost value of the 4-tuple of measurements to the total association number. In the modified method, sparsity $s_{\tau}$ is defined as the ratio of the number of association having passed the gating of the rough correlation test to the number of the fully association. In order to remove some false location points whose association cost are smaller relatively, $s_{\tau}$ is adopted as lower than $s_{0}$ or nearer to it. This can decrease the system error of the model of assignment problem. Therefore, the accuracy of data association of multi-sensor multi-target can be improved correspondingly. 
The experimental function $\tau$ which is given above is just be taken as a approximate superior gating, we can also adjust the size of the gating by observing the number of cost function in the pages of two-dimension at different circumstance timely. In fact, sparsity of candidate target in 4-D assignment problem is usually lower. According to the current accuracy of direction-finding cross location technique, the ratio of the number of cost function need to be calculated to the total number of cost function is about $10-15 \%$ in the two-dimension pages of association cost.

From above analysis, the modified algorithm can be regarded as a two-stage correlation process constituted by both rough correlation process based on verdict function and the accurate correlation process based on the optimal assignment.

\section{Performance of Simulation}

\subsection{Simulation Model}

Suppose the sensors' position are shown in Fig.1, the position of four sensors are ($2000,0),(-1000,-1000 \sqrt{3}),(1000,-1000 \sqrt{3})$ and $(2000,0)$. The bearing measurement error of various sensor is the same value, we use them, $\sigma_{\theta}$ of $0.4^{0}, 0.8^{0}$. The detection probability of each sensor is assumed to be 1 , and the false alarm rate is assumed $1 / \mathrm{rad}$.

\subsection{Analysis of Simulation Results}

As shown in table 1, in the modified algorithm, either the sparsity $s_{\tau}$ is adopted as $s_{\tau} \approx s_{0}$ or adopted as $s_{\tau}<s_{0}$, both the run times reduced largely compared with the former algorithm. This is because that a large quantity of calculation from the false location points is cut. The difference between the two case is that the procedure time of $s_{\tau}<s_{0}$ is little lower than the result of the case of $s_{\tau} \approx s_{0}$. In addition, an obvious result is that the association accuracy correlated to $s_{\tau}<s_{0}$ is advantage of the result of $s_{\tau} \approx s_{0}$. This result mainly because that with the appropriate limitation of the gating of verdict function in the case of $s_{\tau}<s_{0}$, some false location points with lower cost value are removed from the candidate association, and this causes the accuracy of data association and location to be improved by $7 \sim 8$ percents.

As the measurement error is supposed $0.02^{0}$, the relations between the proceeding time of 4-D assignment algorithm and the number of targets are given in Fig.3. It is obvious that the run time growths rapidly with the increase of the number of targets when the former algorithm is adopted, but it increases slowly when the modified algorithm $\left(s_{\tau}<s_{0}\right)$ is used. This result shows that the more the number of targets is, the more obvious the advantage of the modified algorithm demonstrates. In other words, the new algorithm is more applicable to solve the data association problem in high density environment of targets and false alarms. 
Table 1. Average results (25 runs) of 4-D assignment algorithmin (number of targets $=20$ )

\begin{tabular}{cccccc}
\hline & & $\begin{array}{c}\text { Number joining } \\
\text { accurate process }\end{array}$ & $\begin{array}{l}\text { association } \\
\text { accuracy }\end{array}$ & $\begin{array}{l}\text { Run time } \\
(\mathrm{s})\end{array}$ & $\begin{array}{l}\text { RMS error } \\
(\mathrm{km})\end{array}$ \\
\hline $0.4^{0}$ & Former & 1405 & $85.7 \%$ & 75.37 & 1.61 \\
& Later $\left(s_{\tau} \approx s_{0}\right)$ & 1386 & $86.2 \%$ & 33.72 & 1.56 \\
& Later $\left(s_{\tau}<s_{0}\right)$ & 715 & $93.1 \%$ & 31.96 & 1.07 \\
$0.8^{0}$ & former & 4776 & $76.7 \%$ & 84.58 & 2.07 \\
& Later $\left(s_{\tau} \approx s_{0}\right)$ & 4765 & $77.4 \%$ & 37.63 & 2.03 \\
& Later $\left(s_{\tau}<s_{0}\right)$ & 2974 & $85.6 \%$ & 34.41 & 1.58 \\
\hline
\end{tabular}

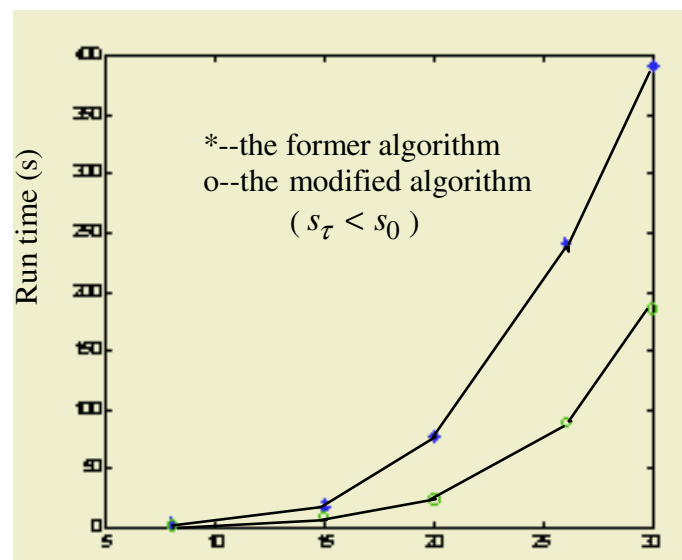

Fig. 3. Run time of 4-Dassignment algorithm versus number of targets

\section{Conclusions}

This text mainly studies data association algorithm in multi-sensor multi-target location system. A modified algorithm based on Lagarangian relaxation algorithm of 4-D assignment problem is presented. The gating of the verdict function in the first stage correlation process is discussed and given. The new algorithm reduces the run time of the global optimal algorithm of 4-D assignment problem, and improves the effect of data association and location. This idea can be developed to the situation that the sensors and targets are not in the same plane, and it has widely application in other fields of the social activities. 


\section{References}

1. Pattipati, S.Deb, K.R, and Bar-shalom, Y. et al.: A new Relaxation Algorithm and Passive Sensor Data Association. IEEE Transactions on Automatic Control, 37(1992), 198-213

2. Yeddanapudi, S.Deb, M. Pattipati, K. Bar-shalom, Y.: A Generalized S-D Assignment Algorithm for Multisensor-Multitarget State Estimation. IEEE Transactions on Aerospace and Electronic Systems, 33(1997) 523-537

3. Jianjuan, X.: Study on Multi-target Algorithm in Two Direction-finding Location System. Acta Electronic Sinica, Beijing, China 30, 12 (2002) 1763-1767

4. Bertsekas, D P.: The Auction Algorithm: A Distributed Relaxation method for the Assignment Problem. Annals of Operat Res, 14 (1988) 105-123

5. You, H.: Multisensor Information Fusion with Application.Publishing House of Electronics Industry. Beijing, China (2000)

6. Wenxun, X.: The Modern optimal Algorithm. Publishing House of Tsinghua University, Beijing, China (1999) 20-28

7. Deb S, Pattipati K.R, and Bar-shalom,Y.: A Multisensor-Multitarget Data Association Algorithm for Heterogeneous sensors. IEEE Transactions on Aerospace and Electronic Systems, 29, 2 (1993) 560-568 